\title{
Sensorless Tension Control in Paper Machines
}

\author{
M. Aníbal Valenzuela, Senior Member, IEEE, John Martin Bentley, Life Fellow, IEEE, and \\ Robert D. Lorenz, Fellow, IEEE
}

\begin{abstract}
Proper operation of the dry end of a paper machine requires some type of sheet tension control. Present systems use either indirect-speed-control-based schemes, or sensor-based direct tension control utilizing load cells or swing rolls. The indirect schemes are inadequate for most newer machines, and both types of the commonly used sensor-based schemes are invasive, expensive, require either special, or extra rolls, and add complexity to the sheet runs.

This paper proposes and evaluates sheet tension estimation using only conventional motor and control signals, present in all newer drive controllers, integrated into a process model observer. This observer-based approach eliminates the need for the invasive tension transducers, and may also be used to replace the inaccurate indirect speed control schemes.
\end{abstract}

Index Terms-Paper machines (PMs), sensorless tension control, tension control, web machine tension control.

\section{INTRODUCTION}

\section{A. Sheet Tension Fundamentals}

A $\mathrm{S}$ THE SHEET is formed and leaves the presses at perhaps $30 \%-40 \%$ water, the sheet has essentially zero modulus of elasticity, and as an inelastic medium, cannot support any stress-producing tension (the reason for closed draws at wet-end sections). As the sheet progresses though the dryer sections, the sheet modulus of elasticity dramatically increases, section-to-section, to higher values as it reaches the final water content of perhaps 5\% (air dry) at the paper machine (PM) reel. Air-dry modulus of elasticity values range from perhaps 400000 $\mathrm{lbf} / \mathrm{in}^{2}$ for newsprint, to over $1000000 \mathrm{lbf} / \mathrm{in}^{2}$ for high-quality bond sheets. It can be readily seen that the water content is the predominant factor determining the sheet modulus of elasticity in the machine direction. Without going through the math involving the sheet caliper (thickness), the sheet modulus of elasticity determines the lineal sheet tension in pound force per lineal inch (pli) for a given steady-state speed difference, regardless of the physical sheet length of a given draw span. This relationship is given by the following generalized per-unit equation:

$$
\begin{aligned}
\Delta \text { Tens } & =k * \Delta N \\
k & =E * t
\end{aligned}
$$

Paper PID 02-37, presented at the 2002 IEEE Pulp and Paper Industry Conference, Toronto, ON, Canada, June 17-22, and approved for publication in the IEEE TRAnSactions on IndUSTRY ApPlications by the Pulp and Paper Industry Committee of the IEEE Industry Applications Society. Manuscript submitted for review June 21, 2002 and released for publication January 7, 2003.

M. A. Valenzuela is with the Department of Electrical Engineering, University of Concepción, Concepción, Chile (e-mail: avalenz@die.udec.cl).

J. M. Bentley is at 6239 Clearview Drive, Manitowish Waters, WI 54545 USA.

R. D. Lorenz is with the Departments of Mechanical Engineering and Electrical and Computer Engineering, University of Wisconsin, Madison, WI 53706 USA (e-mail: lorenz@engr.wisc.edu).

Digital Object Identifier 10.1109/TIA.2003.809449
TABLE I

\begin{tabular}{|c|c|c|c|c|}
\hline Property & News & Fine & LWC & Liner \\
\hline Basis Wt $\left(\mathrm{Lb} / 3000 \mathrm{ft}^{2)}\right.$ & 29.4 & 35.7 & 34.8 & 211.8 \\
\hline Caliper (in) & 0.003 & 0.003 & 0.002 & 0.022 \\
\hline $\begin{array}{l}\text { Modulus of Elasticity } \\
\left(\times 10^{3} \mathrm{lb} / \mathrm{in}^{2}\right)\end{array}$ & $\begin{array}{l}350 / \\
650\end{array}$ & $\begin{array}{l}860 / \\
1250\end{array}$ & $\begin{array}{c}1240 / \\
1450\end{array}$ & $\begin{array}{c}3931 \\
742\end{array}$ \\
\hline $\begin{array}{l}\text { Tensile Strength (Ulti- } \\
\text { mate) (Lb/linear in (pli)) }\end{array}$ & $9.9 / 10$ & $27 / 29$ & $16 / 21$ & $51 / 135$ \\
\hline
\end{tabular}

TENSION-RELATED SHEET CHARACTERISTICS

where

$\Delta$ Tens change in tension (p.u.);

$E \quad$ sheet modulus of elasticity in the machine direction;

$t \quad$ sheet thickness (caliper);

$\Delta N \quad$ speed difference between sections (draw) (p.u.).

Table I summarizes typical salient tension-related characteristics for the most common grades of sheet and board [1].

A guideline for the most probable operating tension for a given sheet grade may be developed from this table, combined with utilizing papermaking experience in setting up a machine direction tension profile through the machine dry-end sections. Experience has shown that it is advisable to limit the section-to-section tension to perhaps no more than $10 \%$ of the tensile strength (ultimate breaking tension). This wide margin is needed to accommodate imperfect paper making conditions, such as "across the sheet" variations in moisture, caliper, etc., producing nonlinear sheet tension "across the sheet."

\section{B. Tension Control Strategies}

1) Speed Difference Indirect Tension Control: Since, for most paper grades, the slope of the stress-strain curve (the modulus of elasticity) is quite linear in the practical operating range as the stress increases up toward the fracture point, we get very predictable tension changes for a given speed difference between sections, if the moisture content remains constant. What is the significance of all this? Common sense prevails, and if the sheet moisture remains nearly constant at a given draw in the dry-end of the PM, a good precise speed controller should provide an excellent indirect method of tension control! We might conclude that a quality speed controller solves most of the problems in determining tension, since it inherently provides inertia compensation during dynamic speed changes as well as friction loss compensation, under all conditions in all elements of the drive train and load, since it theoretically is controlling the peripheral speed of the tension producing draw rolls.

Why does not speed control always do the job? Several factors deteriorate the performance of the speed controller as an indirect tension controller. 
1) The speed controller presumes the relationship between the speed-sensing tachometer and the peripheral velocity of the draw-producing roll(s) remains fixed and linear. This is not achievable, since the indrive train is mechanically imperfect and elastic, and the dimensional periphery of the draw-producing roll(s) is a function of wear, elastic compressibility or distortion, and thermal expansion. These factors, combined with the steady-state accuracy (error) of the speed controller itself (perhaps $\pm 0.1 \%$-analog to $\pm 0.01 \%$-digital), yield unacceptable tension variations on certain grades. For example, on most grades of air-dry wood-free publication paper, by applying (1) we find that the sheet modulus of elasticity and caliper combined, produce about a $10 \%$ tension change for each $0.1 \%$ draw speed difference.

2) The most significant sheet parameter in determining the resulting tension in a draw is the sheet modulus of elasticity in the machine direction. Even if the speed control were totally free from imperfections as discussed above, sheet modulus of elasticity changes would, in most instances, produce intolerable tension changes during paper making transient changes if no adjustments were made to the speed set points at the draw points in the machine. Online speed set-point control is generally available via the process control system to alleviate this situation during machine grade changes, or other "upsets" during machine paper-making operation. Dryer section differential temperature controllers are vital in maintaining the amount of sheet moisture removed for a given section of dryers; therefore, the modulus of elasticity is also indirectly controlled through that section to a certain degree. There are other programmable process changes that are used to initiate tension or speed control set-point manipulation to accommodate large changes in sheet modulus of elasticity. For example, on-machine coaters and size presses are threaded with reduced nip on the dry sheet, then when the sheet is full width and the dryer moisture levels are somewhat stabilized, the coating heads are engaged and suddenly the sheet is rewetted and its modulus of elasticity drastically drops. The draw span at that point would see a drastic reduction in tension if only pure speed difference control was used. On the other hand, if only tension control were used, the resulting drop in modulus of elasticity would result in a drop in sheet tension, then the tension controller would attempt to maintain the set point for the dry sheet tension value, resulting in a sheet break since the wetted sheet could not sustain the tension of the dry sheet set point. Regardless of the type of control used, feedforward information must be derived from the process controller on the coating process, to set the draw tension to the acceptable value for the wetted sheet just as it appears in the draw span. This type of dynamic change in modulus of elasticity generally cannot be tolerated via feedback only from either speed or tension sensor controllers due to the very short transport delay of the sheet passing through the influence of the open draw span. The resulting transient error in the differential speed change in the affected draw span will be too great unless an an- ticipatory decoupling signal is derived from the process control.

3) Tension control by means of speed/draw control presumes that the tension in the sheet may be independently determined, or isolated, between each indrive point. In a succession of dryer sections, this is not always true, and the combination of sheet shrinkage and some small slippage throughout the dryer nest of rolls (not an ideal single nip point), will produce enough error to destroy the ability of the pure speed regulator to indirectly control the actual tension in the sheet into or out of that section.

2) Speed With Torque Limit Tension Indirect Control: Due to the limitations of speed control as outlined above, solutions have evolved from simple variable-ratio position control of sections via a lineshaft drive, through precision digital speed-controlled sectional drives with speed loops to include a current (electromagnetic torque) limit as an indirect form of tension control. This indirect approach also failed to achieve totally acceptable tension control. Torque control schemes have failed to produce the required tension control precision due to the fact that the torque component of the motor input at lighter tension values may be extremely small when compared to the normal running friction load of the section being controlled. For example, on a 12-72-in roll dryer section, with two felts and two doctors, the Technical Association of the Pulp and Paper Industry (TAPPI) power constants yield the following.

$$
\begin{aligned}
& \mathrm{NRL}=0.684 \mathrm{hp} / \mathrm{in} / 100 \mathrm{ft} / \mathrm{min} . \\
& \mathrm{RDC}=0.1764 \mathrm{hp} / \mathrm{in} / 100 \mathrm{ft} / \mathrm{min} . \\
& \text { Sheet Tens Load }=0.003 \mathrm{hp} / \mathrm{in} / 100 \mathrm{ft} / \mathrm{min} / \mathrm{pli} \text { tens. } \\
& \text { Converting to relative torque units for the ratio: } \\
& \text { (Tens torque/NRL Torque) } \times 100 \simeq 5 \% \text { per } 1.0 \mathrm{pli} \text {. }
\end{aligned}
$$

This example shows that each pli of differential sheet tension requires only $5 \%$ of the TAPPI normal running load torque; therefore, the indrive torques responsible for tension will only be a few percent greater than the NRL torques, making it extremely difficult to compute tension from motor loading using conventional control strategies involving motor amperes or motor-algorithm-derived torque values. In the actual multiple dryer machine, several dryer sections like this example are used, and for any given section, only the differential in sheet tension between the upstream draw and the downstream draw manifests itself in the drive motor output torque. This may further reduce the tension producing incremental torque required by the drive, thus making indirect tension control even less precise and, thus, unacceptable.

3) Tension Control by Utilization of Tension Sensors: A great improvement in control of tension may be achieved by adding an outer tension control loop to the speed controlled sectional drives. Two common types of direct tension sensing have been employed in these applications.

1) Swing rolls (position sensors, precision pressure regulators, and low-friction swing arm mechanisms)_A swing roll, in its basic implementation, rotates freely in contact with the sheet and can move approximately perpendicular to the sheet direction according to the force balance between the sheet tension and a restraining pneumatic loading system which is proportional to the desired 


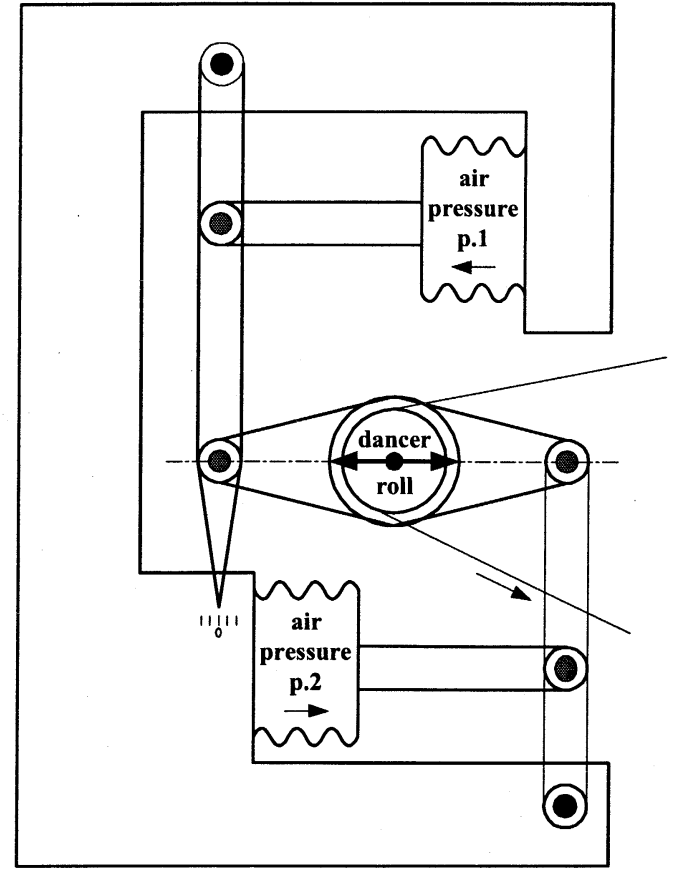

Fig. 1. Typical swing roll arrangement.

tension (Fig. 1). If the sheet tension does not match the regulated pneumatic loading, the swing roll will accelerate to a new position creating an error signal to restore the initial position via a corrective section speed change. In some slower machines producing heavyweight sheets, this was accomplished via a simple dancer roll counterbalanced with weights. The main advantage claimed for the swing roll system applied to paper making machines is the sheet storage provided during transient upsets such as the transition from a dry sheet to a coated sheet. This same principle, also presents one of the major technical disadvantages of swing roll control, especially when adjacent swing roll sections are involved such as in two side coating. The two sections, plus the associated dryer sections, interact to the sheet storage and a very complex system is needed to decouple them and provide the stability required. The position sensor is very inexpensive, simple, and drift/noise free, as compared to the electronic technology required by the load cell system.

The pressure regulation would seem to be a critical element in the force calibration. Also, the actual tension signal desired would be directly related to the acceleration of the dancer roller, not the position deviation resulting from acceleration. Closing a loop on the position deviation will assure steady state tension properties, assuming pressure regulation is ideal.

2) Force transducers (load cells and low-inertia tension rolls)—A load cell tension roll, in its basic implementation, uses a roll mounted on force-sensing load cells (Fig. 2). Two different types of load cells are most commonly used in PM applications: variable permeability load cells, and cross-coil load cells. Depending on the mounting position of the roll, the weight of the roll can be neglected or must be compensated in the output

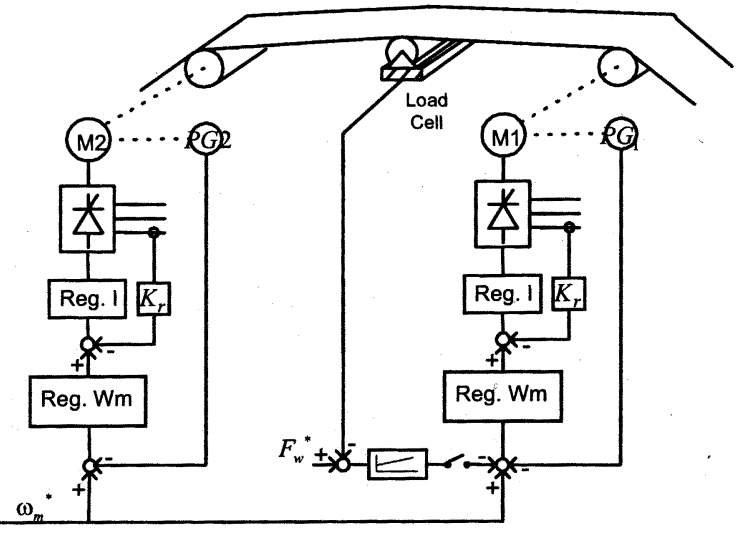

Fig. 2. Tension control using load cell.

signal. Usually, the output signal needs some filtering to eliminate noise caused by mechanical unbalance and vibration. The main advantages of load cells are their cost when compared to swing rolls, adaptability to most sheet runs where the load cell roll may be used to implement the sheet run geometry, and essentially zero sheet length storage. They are however very sensitive to proper alignment and balance in higher speed machines. If a drive is required to accommodate use of this roll for threading, or stopping, the indrive shafting must be provided with a double bearing and flexible coupling to isolate the roll from lateral forces from the drive motor or reducer. These tension rolls, by their very nature are very "fragile" compared to other rolls of a PM; consequently require additional maintenance, both electrically and mechanically. On higher speed machines the balance may be so critical that the roll must be kept rotating at all times when the PM is down, or they will distort thermally due to the radiated heat from an adjacent dryer can. In addition, on all but the slowest and narrowest machines, two-ended sensing is required to obtain a reasonable across the sheet average for tension computation. This necessitates that both ends of the roll be equipped with special bearing pillow blocks and load cells, resulting in additional initial costs and on-going maintenance.

Ebler et al. [2] evaluated both tension sensors in regard to their application to PM drives. Their analysis considered tension control system stability and dynamic response. They concluded that, for all practical purposes, there is no difference in implementing tension control using swing rolls or load cells. However, if mounting conditions favor minimal filtering, then load cell tension control is superior.

Nevertheless, both options are invasive (i.e., they need a physical contact with the sheet to sense the tension), expensive, require additional machine space, and need adjustment. Therefore, implementing tension control without tension sensors would be beneficial from an economic standpoint.

4) Sensorless Tension Control: Based on sheet physics, tension at any draw span of the PM will reflect as a torque component that will add to the load torque of the downstream drive, and will subtract from the load torque of upstream drive. Therefore, a sheet tension observer could be implemented based on the drive torque components, related to the tension in 


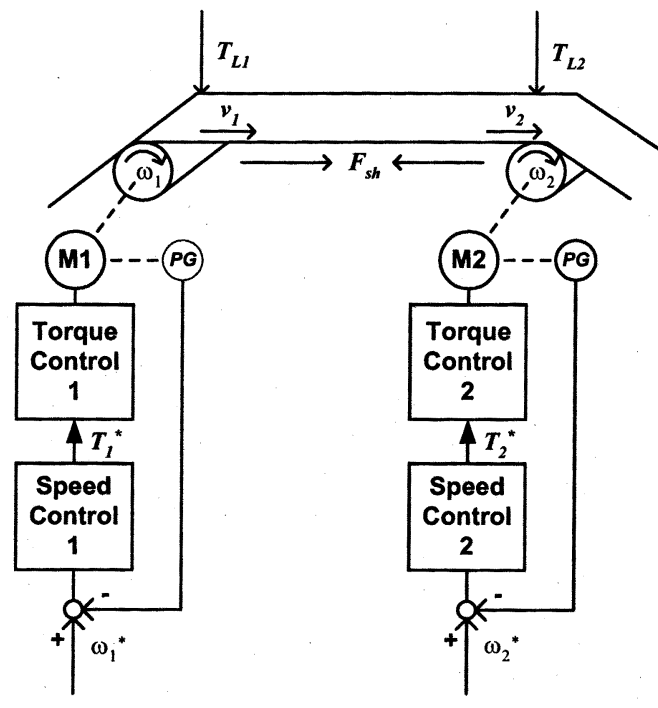

Fig. 3. Two-section system.

the draw span. This estimator would be dynamic and provide constantly updated observer computation of the sheet tension between drives, providing superior performance to the existing sensorless schemes involving speed control with motor torque limiting or speed droop as a function of motor torque.

As indicated above, there are several technical shortcomings of any known system used to control draw span tension. It will be the initial objective of this paper and associated laboratory work, to develop a tension estimator based control system that will yield performance that is comparable, or better, than those systems currently used on PM sections, and eliminate the need for sheet tension sensors.

\section{Two-Section System Modeling AND Evaluation}

The fundamentals of tension estimation in an elastic sheet will be first developed in a two-section system. Then, in the following section, this sheet tension algorithm will be used in the evaluation of a three-section system consisting of the last three sections of a high-speed lightweight PM.

\section{A. System Modeling}

Fig. 3 shows a two-section drive system transporting a sheet. It is assumed that no slip occurs between the rolls and the paper sheet, and that the sheet is elastic. Fig. 4 shows the block diagram of the two-section system including the effect of the sheet tension on the drives. The dynamic equations are

$$
\begin{aligned}
& T_{1}=J_{1} \bullet \frac{d \omega_{1}}{d t}+T_{L 1}-r_{1} \bullet F_{\mathrm{sh}} \\
& T_{2}=J_{2} \bullet \frac{d \omega_{2}}{d t}+T_{L 2}+r_{2} \bullet F_{\mathrm{sh}} .
\end{aligned}
$$

For an elastic paper sheet of negligible mass, the sheet tension is proportional to the stretch of the sheet, and can be expressed as

$$
F_{\mathrm{sh}}=K_{\mathrm{sh}} \int\left(r_{2} \omega_{1}-r_{1} \omega_{2}\right) \bullet d t=K_{\mathrm{sh}}\left(r_{2} \theta_{1}-r_{1} \theta_{2}\right)
$$

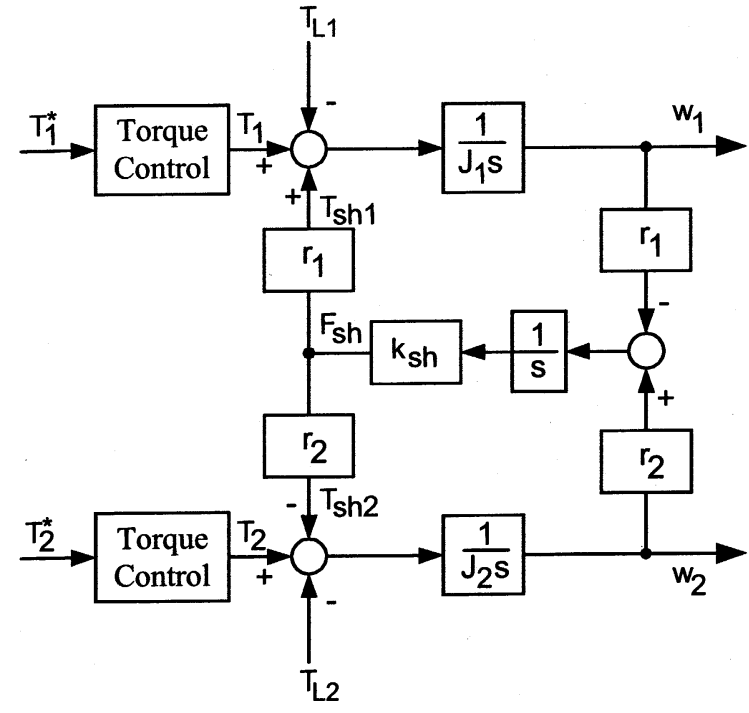

Fig. 4. Two-section elastic sheet tension. model.

where $K_{\mathrm{sh}}$ is the (assumed) elastic stiffness of the sheet which depends on the paper grade and the process operating conditions. For a specific paper grade the main process variable that affects $K_{\mathrm{sh}}$ is the moisture content. As explained in the introduction, if the PM runs under stable process and drive operating conditions, it can be assumed that $K_{\mathrm{sh}}$ at each dry-end section remains constant.

Under constant-speed operating conditions, the inertial torque is zero, and the electromagnetic torque developed by the drives is that obtained from simple static, freebody analysis

$$
\begin{aligned}
& T_{1, c s}=T_{L 1}-r_{1} \bullet F_{\mathrm{sh}} \\
& T_{2, c s}=T_{L 2}+r_{2} \bullet F_{\mathrm{sh}} .
\end{aligned}
$$

The constant-speed electromagnetic torque components can be obtained from the torque commands in vector controlled or direct-torque-controlled ac drives or from the armature currents of dc drives. In order to estimate the sheet tension there is a need to separate the sheet tension component and the load torque (bearing friction and machine windage) component of the developed torques. This can be done using the fact that the sheet tension torque component is present in the two drives in the opposite sense.

Therefore, if the system is running at constant speed and constant load with the paper sheet without slack or tension, then the developed torques reduce to

$$
\begin{aligned}
& T_{1,0}=T_{L 1} \\
& T_{2,0}=T_{L 2}
\end{aligned}
$$

with related torque-producing currents $I_{a 1,0}$ and $I_{a 2,0}$.

If from this initial condition the speed of the downstream section is lightly increased, the sheet starts to stretch and tension torque components will show up, increasing the torque (and current) of drive 2, and decreasing the torque (and current) of drive 


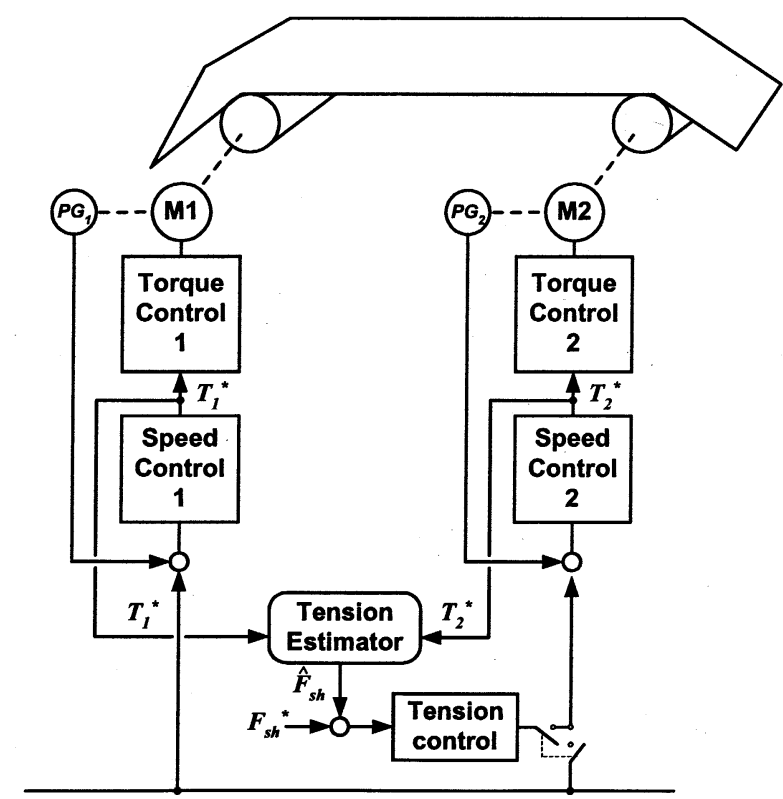

Fig. 5. Tension estimation (and control) using torque command signals.

1. The resulting sheet tension can be calculated from the variation of the currents as

$$
\begin{aligned}
& F_{\mathrm{sh}}=-\frac{T_{1, c s}-T_{1,0}}{r_{1}}=K_{m 1} \bullet \frac{-\Delta I_{a 1}}{r_{1}} \\
& F_{\mathrm{sh}}=\frac{T_{2, c s}-T_{2,0}}{r_{2}}=K_{m 2} \bullet \frac{\Delta I_{a 2}}{r_{2}} .
\end{aligned}
$$

The proposed sheet tension estimation algorithm for a drive system operating at constant speed is based on these two equations. Both expressions can be computed independently and must give the same results, which will confirm the validity of the estimated value. It should be noted that this basic estimator for drives running at constant speed can readily be extended to become a dynamic estimator by including the inertial torque terms, i.e., forming an open-loop observer, and adding a feedback controller, i.e., becoming a closed-loop observer. Such a closed-loop observer would be necessary for variable-speed operating equipment, like winders and coaters.

An open-loop observer with feedforward inputs of $T_{1}^{*}$ and $T_{1}^{*}$ and outputs of the estimated states $\hat{\omega}_{1}, \hat{\omega}_{2}$, and $\hat{F}_{\mathrm{sh}}$ would be the same as the tension model without the $T_{L 1}$ and $T_{L 2}$ disturbance inputs since they are not known. If constant values of $T_{L 1}$ and $T_{L 2}$ are used, the system will have the same static properties as the simplified steady-state model. Since no damping is included in the model, it would be an oscillator, which would be highly undesirable.

A closed-loop observer with feedforward inputs of $T_{1}^{*}$ and $T_{1}^{*}$, measured state references of $\omega_{1}$ and $\omega_{2}$, and outputs of the estimated states $\hat{\omega}_{1}, \hat{\omega}_{2}$, and $\hat{F}_{\mathrm{sh}}$ as well as estimated disturbance inputs of $\hat{T}_{L 1}$ and $\hat{T}_{L 2}$ would be more desirable. The controller would allow damping of the observer poles. With this model, only two independent output states could be estimated. If one output state is $\hat{F}_{\mathrm{sh}}$, then the second could be the average velocity.

\section{B. Two-Section System Evaluation}

The proposed tension estimator is shown in Fig. 5 for the two-section system. During the startup, both drives are independently accelerated to the operating (or threading) speed, and the rolls attached to them remain running at the same linear velocity, waiting for the paper sheet to be threaded.

After the paper sheet is passed through the two sections, some sheet slack usually is present in the span between the sections. The operator, using the slack-take-up signal, eliminates the sheet slack, and the system will be ready for tension control.

When tension control is activated, the tension estimation block measures and computes the torque component currents corresponding to the section load torques using (8) and (9). Then, the tension control system applies an auxiliary signal to the section 2 reference in order to increase its speed and start stretching the paper sheet. This signal is applied until the torque signals in drives 1 and 2 match the values corresponding to the desired sheet tension using (10) and (11).

Fig. 6 simulates this sequence. For sake of simplicity, twin drives were chosen for both sections, both of them operating with identical load torques (50\% of nominal torque), and driving rolls of equal diameters. The initial amount of sheet accumulated in the span is removed by the operator applying step 1 , from 1 to $3.2 \mathrm{~s}$. After that, the tension control is activated and a new step (step 2) is applied to set the sheet tension to the desired value. Correspondingly, the armature current of section 2 increases and section 1 decreases, according to the new operating condition.

Once the system is running, and while the load torques and sheet tension remain constant (condition checked by measuring the currents), there is no need of further adjustment. Additionally, the tension estimation algorithm is capable of distinguishing sheet tension changes due to section load changes, and it is possible to test the existing tension in the draws. This can be done by applying a slow changing auxiliary signal to section 2 , while measuring the associated varying currents. Fig. 7 shows the simulated results obtained applying a 1-Hz sinusoidal auxiliary signal, from which it is possible to estimate the sheet tension $F_{\text {sh }}$ according to (10).

\section{EVAluation of THE Dry END OF A PAPER MAChine}

The tension estimation algorithm will now be applied to a more realistic system consisting of the last three sections of a high-speed lightweight PM. This evaluation will show the performance of the proposed Estimation \& Tension Control Block applied to high-inertia drives running with very small differential tension.

The three-section arrangement selected for this evaluation is depicted in Fig. 8. It consists of the last sections of a $2500-\mathrm{ft} / \mathrm{min}$ ( $762 \mathrm{~m} / \mathrm{min}$ ), $34 \mathrm{lb} / 3000 \mathrm{ft}^{2}$ lightweight coated PM (LWC). The dryer section is formed by 1260 -in cans, 12 felt rolls, and two doctors. The calender is assumed to consist of four 24-in-diameter rolls (for simplicity all having the same ID and OD) forming three nips, and one doctor. One roll is a Beloit CC (controlled crown) operating at $600 \mathrm{pli}$. The reel consists of a 42 -inch drum, one doctor, and a full shipping roll of 100-in diameter. 


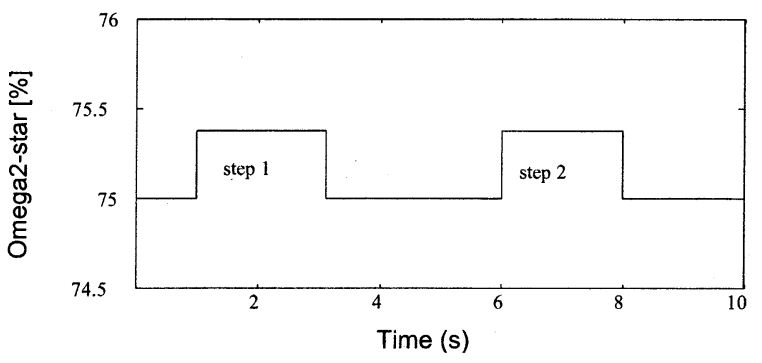

(a)

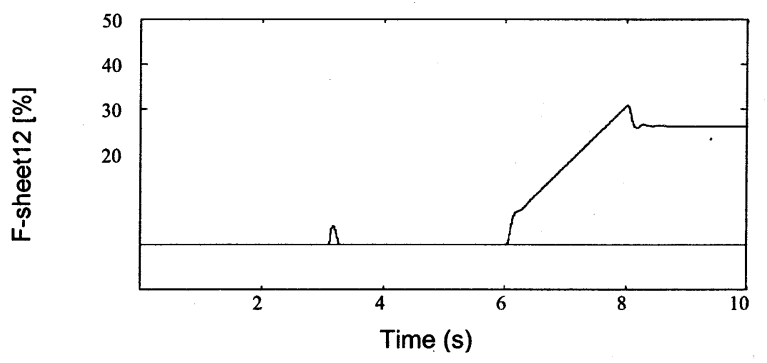

(c)

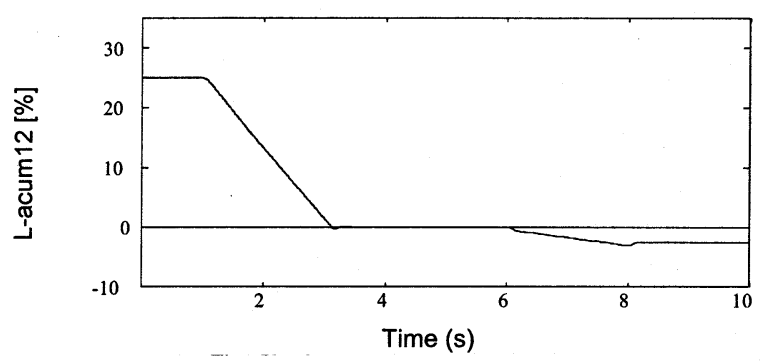

(b)

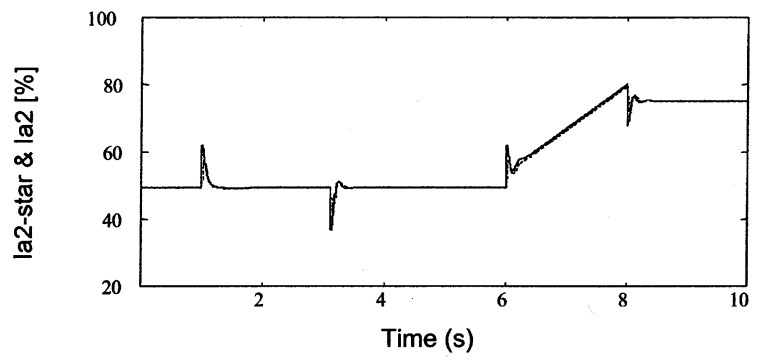

(d)

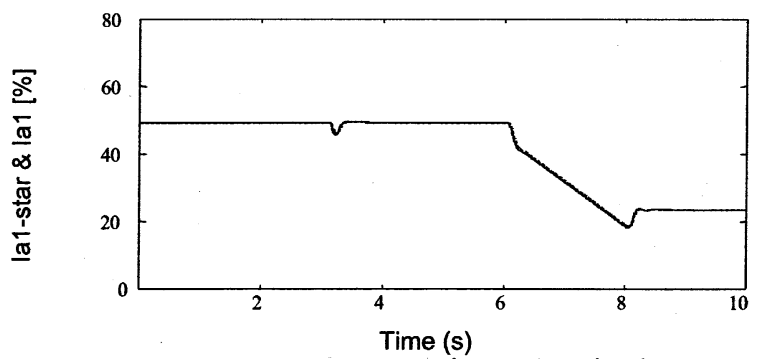

(e)

Fig. 6. Slack-take-up auxiliary signal and setting of operating sheet tension. (a) Velocity reference section 2. (b) Accumulated sheet in span 1-2. (c) Sheet tension in span 1-2. (d) Reference and current section 2. (e) Reference and current section 1.

Drive ratings were selected using TAPPI Recommendations (RDCs). Sensorless tension loops are implemented in the outgoing draw of the last dryer section (traditionally implemented with a load cell tension sensor), and in the ingoing draw of the reel (traditionally implemented as torque-limited indirect tension control).

Running loads and tension references also follow TAPPI Recommendations. Therefore, each section runs initially with its NRL load, and tension references are 1.01 pli in the calender tension loop, and 1.59 pli at the reel tension loop. NRL loads and tension component loads are summarized in Table II.

Initial condition is with the PM running at $2500 \mathrm{ft} / \mathrm{min}(762$ $\mathrm{m} / \mathrm{min}$ ) and sheet in draws 4-5 dryer-calender and 5-6 calender-reel already adjusted so that no paper sheet is accumulated in these draws. Simulation includes $200 \mathrm{~s}$. At $t=10 \mathrm{~s}$, the tension loop of the calender is activated through a 10-s ramp. After that, at $t=60 \mathrm{~s}$, the tension loop of the reel is activated, also using a 10-s ramp. These fast ramps have been selected to reduce the simulation time, and they impose relatively fast transient tension commands to the system.

Fig. 9 shows the waveforms of the variables of interest during these transients. Fig. 9(a) depicts the ramp references applied to the tension loops. Fig. 9(b)-(d) shows the tension torque com- ponents of each section. Fig. 9(c) and (d) also includes the estimated tension torques of the calender (T5-hat) and the reel (T6hat). Finally, Fig.9(e)-(h) shows the associated current waveforms.

Fig. 9(c) and (d) confirms the robustness of the tension estimation algorithms. In both figures, the estimated tension torques T5-hat and T6-hat completely agree with the tension torque components T5-tn and T6-tn, respectively. The only difference is that tension torque components show some level of oscillation right after the activation of the reel tension loop which do not show up in the estimated tension torques.

In addition, Fig. 9(d) shows that some level of tension torque appears in the reel after the activation of the calender tension loop, even though the reel tension loop is still inactive. This tension torque is due to some level of transient stretch of the sheet in the ingoing draw of the reel because of the different transient response of the drives to the cascade draw signal after the calender tension loop activation. This existing tension in the sheet causes a small disturbance when the reel ramp reference is activated. If the initiation of this second ramp is delayed (as it probably occurs in real applications), the previous tension of the sheet in the span 5-6 should vanish, and both loops will be greatly decoupled. 


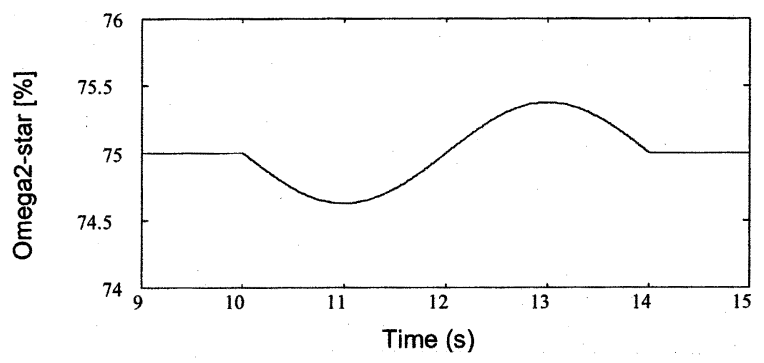

(a)

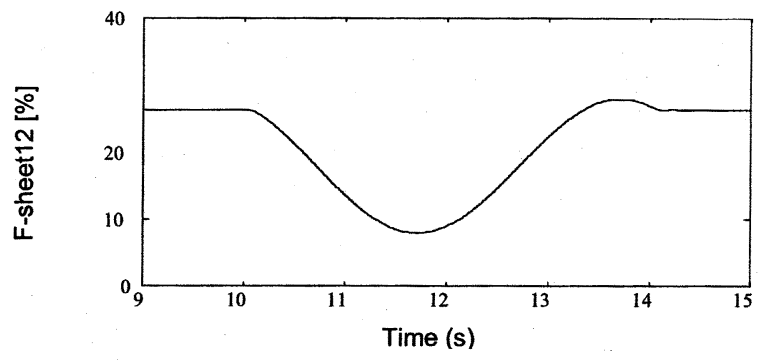

(c)

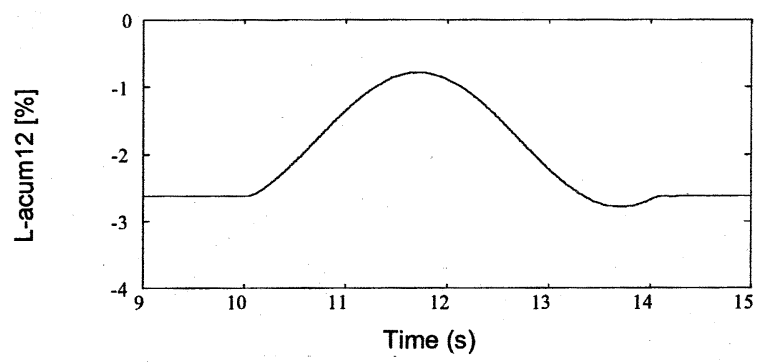

(b)

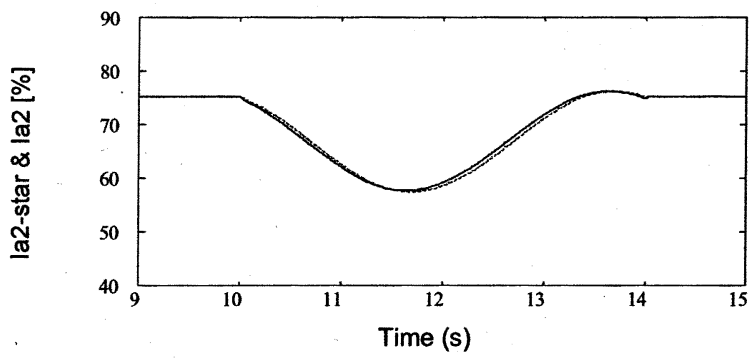

(d)

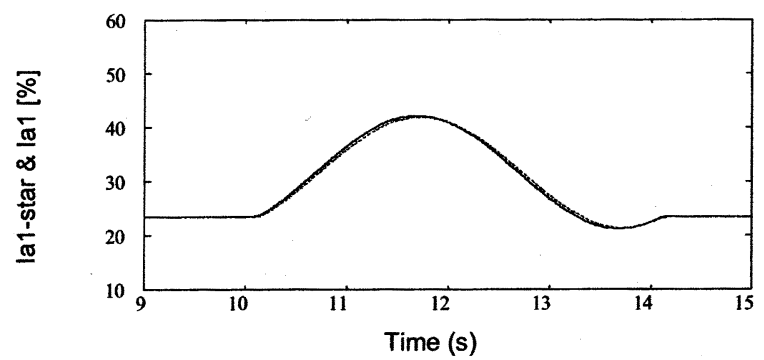

(e)

Fig. 7. Tension estimation using sinusoidal auxiliary reference. (a) Sinusoidal auxiliary signal section 2. (b) Accumulated sheet in span 1-2. (c) Sheet tension in span 1-2. (d) Reference and current section 2. (e) Reference and current section 1.

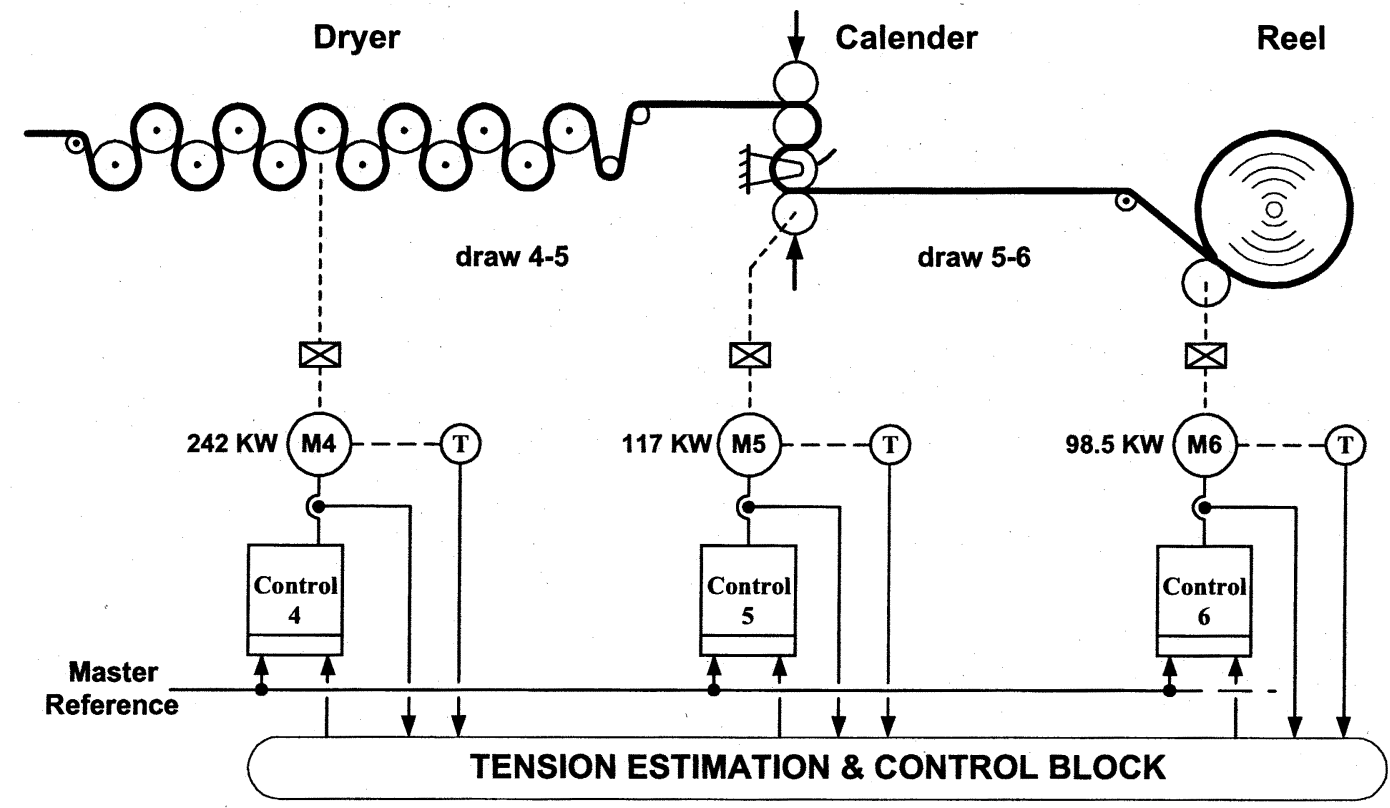

Fig. 8. Dry-end arrangement of a high-speed lightweight PM. 


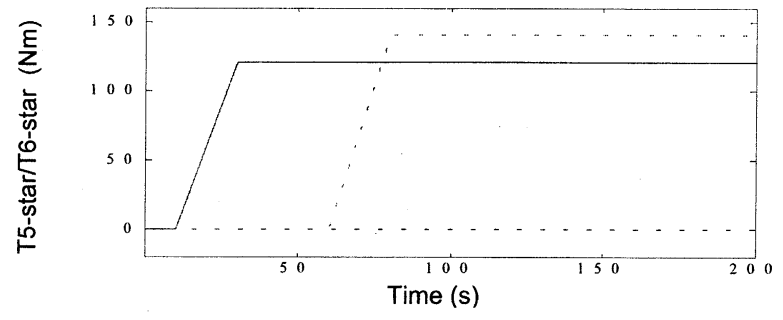

(a)

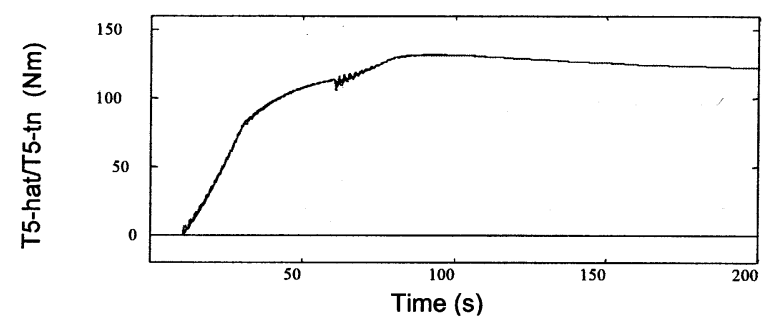

(c)

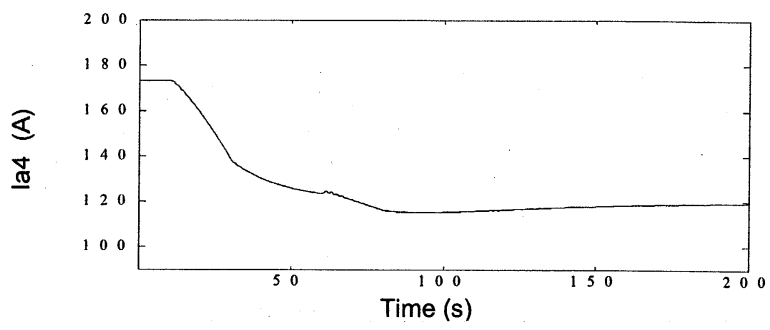

(e)

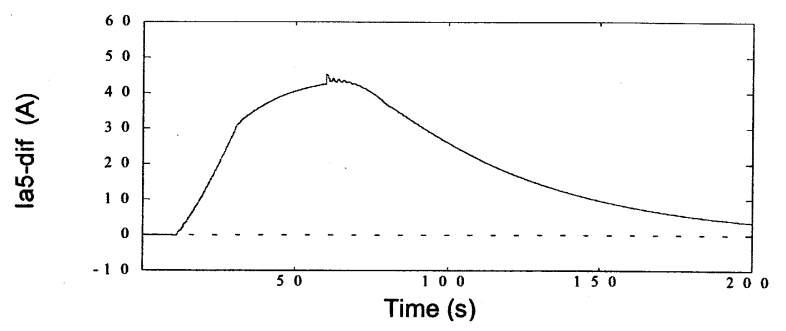

(g)

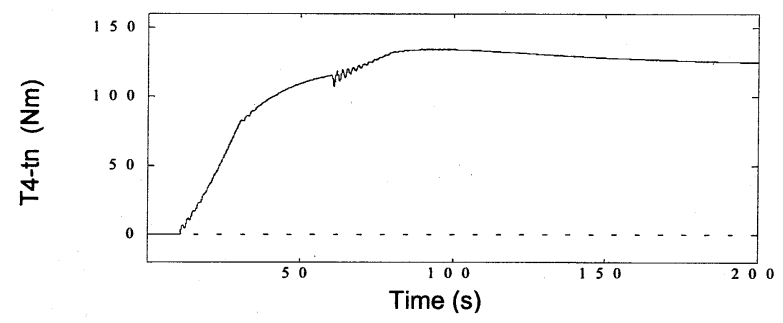

(b)

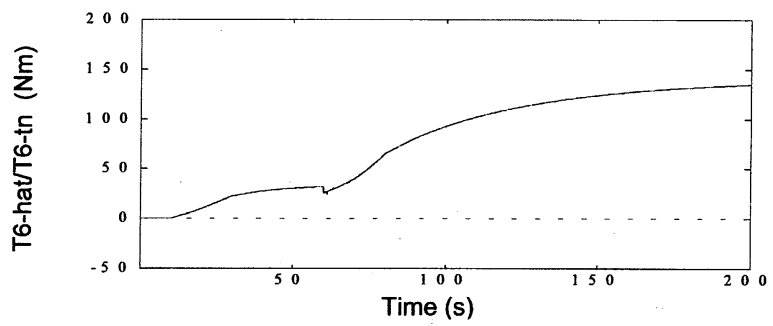

(d)

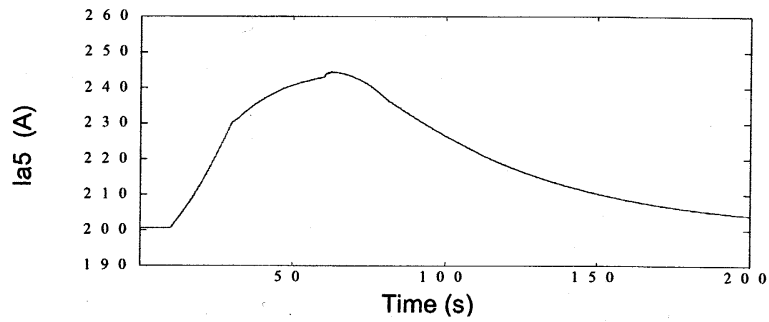

(f)

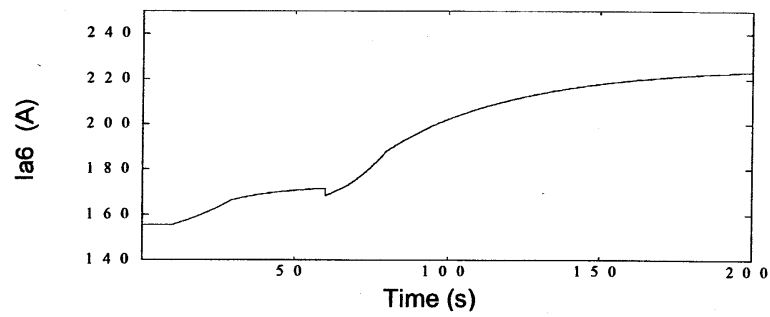

(h)

Fig. 9. Evaluation of tension estimation and tension control in a three-section system of the dry end of a PM (dryer-calender-reel). (a) Tension references of calender and reel sections (drives 5 and 6). (b) Tension torque component in dryer section (drive 4). (c) Tension torque estimation and tension torque component in calender section (drive 5). (d) Tension torque estimation and tension torque component in reel section (drive 6). (e) Current in dryer section (drive 4). (f) Current in calender section (drive 5). (g) Differential current in calender section (drive 5). (h) Current in reel section (drive 6).

TABLE II

NRL AND TENSION POWER COMPONENTS

\begin{tabular}{lcc}
\hline \multicolumn{1}{c}{ Section } & NRL & Tension \\
\hline Dryer & $83,18 \mathrm{KW}$ & $16.15 \mathrm{KW}$ \\
& $(111.54 \mathrm{HP})$ & $(21.66 \mathrm{HP})$ \\
Calender & $85.30 \mathrm{KW}$ & $25.44 \mathrm{KW}$ \\
& $(114.39 \mathrm{HP})$ & $(34.12 \mathrm{HP})$ \\
Reel & $55.83 \mathrm{KW}$ & $25.43 \mathrm{KW}$ \\
& $(74.87 \mathrm{HP})$ & $(34.11 \mathrm{HP})$ \\
\hline
\end{tabular}

Fig. 9(e) shows the evolution of the current in the dryer section. Initial current is about $170 \mathrm{~A}$, which agrees with the NRL torque imposed to the section. After the activation of the calender tension loop, the current in the dryer section starts to reduce going to a new steady-state value of $120 \mathrm{~A}$. Before this happens, at $t=60 \mathrm{~s}$, due to the disturbance produced by the activation of the reel tension loop, the current goes below its steady-state value and then is restored to it.

Fig. 9(f) and (g) illustrates the effect of the ingoing and outgoing sheet tensions on the calender current. Initially, the current is close to $200 \mathrm{~A}$, corresponding to the NRL power. When the calender tension loop is activated, the current in the calender starts to increase due to the tension in span 4-5, eventually getting a new steady state of $260 \mathrm{~A}$, which reflects the $25.44 \mathrm{~kW}$ of tension power set in the tension loop. At $t=60 \mathrm{~s}$, the reel tension loop is activated and the outgoing tension component in draw 5-6 starts to increase and, therefore, the current Ia5 starts to reduce. As the tension reference of the sheet in draw 5-6 is almost equal to the tension reference in draw 4-5, the incoming and outgoing tension components in the Calender tend to cancel their effects and the current Ia5 eventually reaches a 
current level almost equal to its NRL current, with zero differential tension. This effect can be seen in Fig. 9(g) which shows the differential component of the calender current, starting at zero, then going to a maximum value of about $45 \mathrm{~A}$ when only the tension control in span 4-5 is activated, and then reduces its value to almost zero. During this entire operation, the Estimated Tension Block continues to output the correct values of the tension torque components.

As the reel is the end section of the PM, its currents (torque) are only affected by the load of the section, and the sheet tension component in draw 5-6. Therefore, current Ia6 has the same shape as torque T6 [see Fig. 9(d)]. Its initial value of 155 A matches the NRL torque of the section, and after the application of the reel tension reference, its value increases, reaching a final value of $225 \mathrm{~A}$, which matches the $25.43 \mathrm{kKW}(34.11 \mathrm{hp}$ ) set in the tension loop.

In conclusion, these simulation results of the last three sections of a high-speed lightweight coated PM, using real parameters and operating conditions, demonstrate that sensorless tension control can properly handle the practical situations that appear in this application. The constant-speed-based tension estimation algorithm is capable of determining the correct torque tension components, even in central sections where the ingoing and outgoing sheet tension almost cancel, and differential tension goes to zero.

\section{Tension Estimation Using a Low-FreQuency Signal}

As explained in the previous paragraph, the tensions of the sheets in the controlled draws are obtained from the current signals of the involved drives. First, the currents without sheet tension are acquired by the system (and eventually checked with the expected NRL currents). Then, the system monitors current variations in the sections during tension loop activation. All these variations are cross-checked at both ends of each draw to distinguish tension from any load variations in the sections.

This algorithm has shown to be very robust and capable of estimating the tension torque components very accurately. This accuracy may be increased further by inputting process signals to the Estimation \& Tension Control Block. These signals would be related to the section load variations caused by operational functions such as: condensate extraction from the dryer cans, pli pressure adjustment in calender, and diameter of paper roll in the reel.

This control scheme also makes it possible to obtain the existing sheet tension in the different draws through the addition of a low-frequency auxiliary signal to the drives, and measuring its effects in the upstream and downstream sections. This signal can be as low as $2 \%-3 \%$ of the operating current and will reflect in the neighbor sections as a low-frequency tension torque components, which can be extracted using a digital Fourier transform (DFT) algorithm [3], similar to those used to get the harmonics in distorted current waveforms.

This procedure was implemented in the laboratory using an experimental setup consisting on an ac drive and a $\mathrm{dC}$ braking generator feeding a constant load (see Fig. 10). A 3\% 1-Hz sinusoidal signal is added to the armature current of the braking

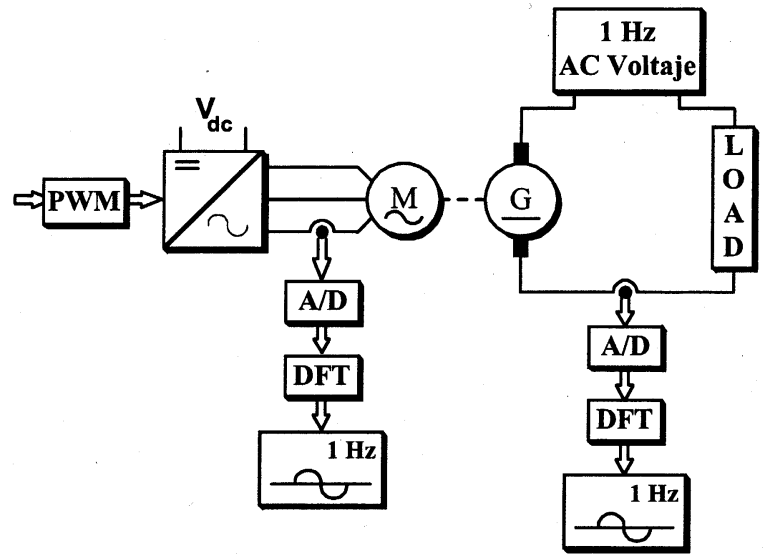

Fig. 10. Experimental setup to generate and extract a low-frequency torque variation.

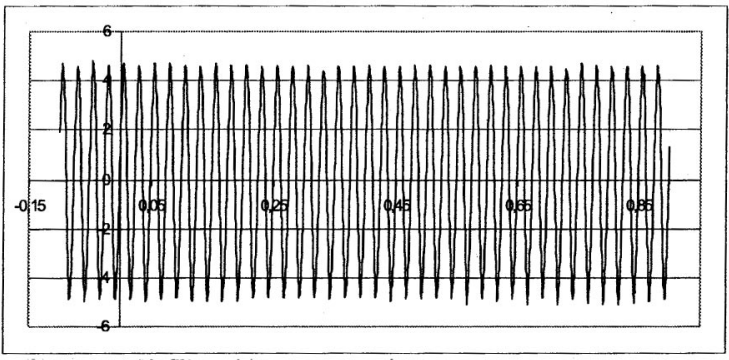

(a)

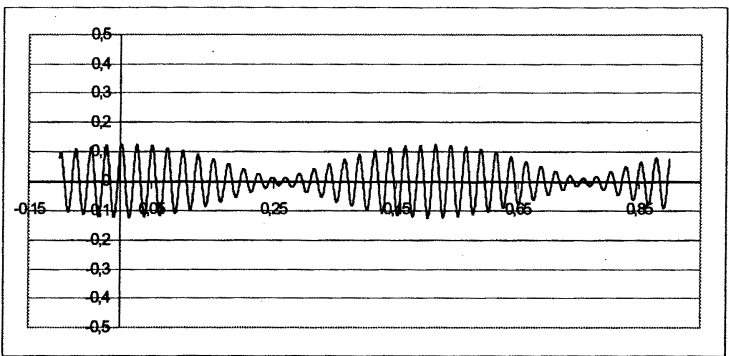

(b)

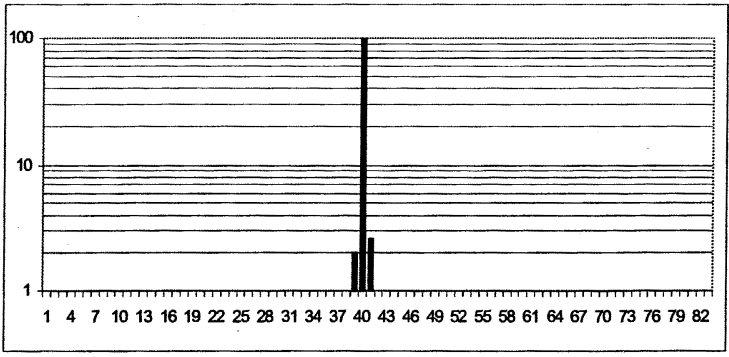

(c)

Fig. 11. Unfiltered waveform, 39- and 41- $\mathrm{Hz}$ components, and frequency spectrum of current including a $3 \% 1-\mathrm{Hz}$ torque variation. (a) Unfiltered current waveform, including low-frequency torque. (b) $39-$ and $41-\mathrm{Hz}$ components. (c) Frequency spectrum.

generator using a sinusoidal signal generator with a high-current output stage. Current signals in both machines are measured, filtered, and finally the $1-\mathrm{Hz}$ components are extracted using the DFT algorithm.

Fig. 11 shows the unfiltered current waveform [Fig. 11(a)], the sum of the 39- and 41-Hz components [Fig. 11(b)], and the 
frequency spectrum of the current [Fig. 11(c)] when the ac drive is fed with a $40-\mathrm{Hz}$ voltage and loaded with a $3 \% 1-\mathrm{Hz}$ varying torque component. The $1-\mathrm{Hz}$ signal related to the varying torque of the ac drive is computed from the $39-$ and $41-\mathrm{Hz}$ components. The extracted $1-\mathrm{Hz}$ component signal pretty well agrees with the varying torque of the dc braking generator.

This experimental setup shows that is also possible to include, in the Estimation \& Tension Control Block, the capability of estimating the sheet tension using this low-frequency injection technique.

\section{CONCLUSIONS}

A new sensorless sheet tension control for the dry end of a PM has been presented and evaluated. This system eliminates the sheet tension sensors, and only uses the same current signals actually required in the speed/torque control of the drives.

The new and novel control element in this system is a tension estimation algorithm, which computes and cross-checks the effect of sheet tension on the current of the drives at both ends of each draw. It is robust to differential tension, and capable of maintaining its accurate estimation, even with zero differential tension. This ability was proved in the evaluation of the sensorless tension control loops implemented in a dryer-calender-reel three-section subsystem of a high-speed lightweight PM.

The work done so far also opens new issues of interest regarding sheet tension control. One of them is the addition of the process signals, related to section load variations, to the Estimation \& Control Block. Even though the tension algorithm has shown good robustness in regard to load variations, utilizing these process based signals would be very desirable in the development of a sensorless tension control for the entire dry end of a PM, which could include up to eight sections.

In addition, as PMs operate at essentially constant speed, the developed tension estimation algorithm described in this paper does not include the inertial torque component. It would be of interest to extend and evaluate the sensorless tension algorithm to variable-speed applications, like winders and coaters.

\section{ACKNOWLEDGMENT}

The authors wish to express their gratitude to Prof. J. Espinosa of the Department of Electrical Engineering, University of Concepción, for his help in the experimental signal measurement and processing.

\section{REFERENCES}

[1] A. Olshansky, "Introduction to roll structure," presented at the 1999 TAPPI Paper Machine Drives Short Course, Atlanta, GA, May 18-20, 1999.
[2] N. A. Ebler, R. Arnason, G. Michaelis, and N. D'Sa, "Tension control: Dancer rolls or load cells," IEEE Trans. Ind. Applicat., vol. 29, pp. 727-739, July/Aug. 1993.

[3] G. F. Franklin, J. D. Powell, and M. Workman, Digital Control of Dynamic Systems. Reading, MA: Addison-Wesley, 1998.

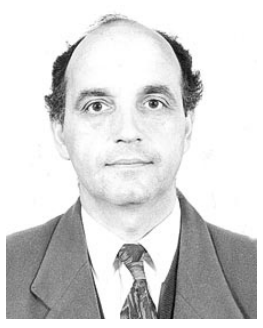

M. Aníbal Valenzuela (M'93-SM'01) received the electrical engineering degree and the Masters degree in electrical engineering from the University of Chile, Santiago, Chile, in 1976 and 1978, respectively.

Since 1978, he has been with the Department of Electrical Engineering, University of Concepción, Concepción, Chile, where he is an Associate Professor in the area of electric machines and drives. His current research interests include motion control of multidrive systems, sensorless control of ac drives, and thermal evaluation of induction motors.

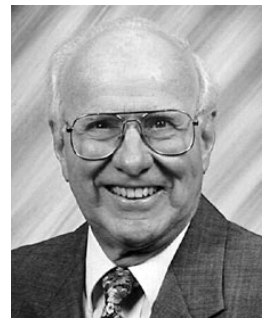

John Martin Bentley (M'54-SM'83-LS'94-LF'99) received the B.S. degree in electrical engineering from Pennsylvania State University, University Park, in 1951.

From 1951 to 1984 , he was with Westinghouse Electric Corporation, Pittsburgh, PA, and Buffalo, NY. He was awarded the company's high engineering position of Fellow, and became recognized as an authority in the field of automatic control and drive systems for the paper industry worldwide, including the first digital sectional and winder drives in North America. From 1984 to 1991, he was Principal Engineer with Allen-Bradley/Stromberg in the Milwaukee, WI, area, and was responsible for in-depth technical and marketing guidance to this multinational joint venture. During this period, he was instrumental in introducing the ac vector drive system to the North American market. Over 1000 sections, totaling approximately $200000 \mathrm{hp}$ were applied on sectional, winder, and finishing equipment drives, including the first sectional ac paper machine drive in North America. From 1991 until his retirement in 1996, he was Principal Engineer with ABB Industrial Systems Inc., New Berlin, WI, and ABB Industry OY, Helsinki, Finland. He provided application and design guidance to the worldwide ABB Pulp and Paper Systems Drives organization, primarily in the area of high-performance ac drive and control systems. He has also been an author and instructor in his areas of expertise for many years. Since his 1996 retirement from $\mathrm{ABB}$, he has continued to consult on a part-time basis, providing expertise in the areas of electromechanical influences on high-performance drive systems, cable and grounding techniques for controlling ac drive system induced common-mode currents, and general guidance for the selection and application of high performance ac drive systems.

Mr. Bentley was recognized for his professional contribution within the Technical Association of the Pulp and Paper Industry (TAPPI) through the TAPPI Engineering Division Award and the E. H. Neese Prize in 1981. A similar honor was acknowledged from the IEEE Industry Applications Society as he was a recipient of the Pulp and Paper Industry Committee Meritorious Achievement Award in 1979. He was appointed to the status of TAPPI Fellow in 1986, and in 1999 was elected as an IEEE Fellow "For contributions to the application and design of electrical drive systems in the pulp and paper industry." The Fellow status is one of the most prestigious honors bestowed by TAPPI and IEEE, and he is the only living member that has been awarded the Fellow status by both organizations. 


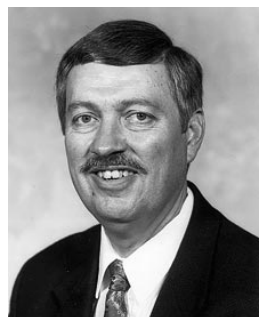

Robert D. Lorenz (S'83-M'84-SM'91-F'98) received the B.S., M.S., and Ph.D. degrees from the University of Wisconsin, Madison, and the M.B.A. degree from the University of Rochester, Rochester, NY.

Since 1984, he has been a member of the faculty of the University of Wisconsin, Madison, where he is the Mead Witter Foundation Consolidated Papers Professor of Controls Engineering in both the Department of Mechanical Engineering and the Department of Electrical and Computer Engineering. He is Co-Director of the Wisconsin Electric Machines and Power Electronics Consortium, which celebrated its 20th anniversary in 2001. It is the largest industrial research consortium on motor drives in the world. He is also the thrust leader for control and sensor integration in the Center for Power Electronic Systems, an NSF Engineering Research Center (ERC) which is a joint ERC with Virgina Polytechnic Institute and State University, Rensselaer Polytechnic Institute, University of Puerto Rico-Mayaguez, and North Carolina A\&T. From 1972 to 1982, he was a member of the research staff at the Gleason Works, Rochester, NY, working principally on high-performance drives and synchronized motion control. He was a Visiting Research Professor in the Electrical Drives Group, Catholic University of Leuven, Leuven, Belgium, in the summer of 1989 and in the Power Electronics and Electrical Drives Institute, Technical University of Aachen, Aachen, Germany, in the summers of 1987, 1991, 1995, 1997, and 1999, where he also was the SEW Eurodrive Guest Professor from September 1, 2000 until July 7, 2001. In 1969-1970, he conducted Master thesis research in adaptive control of machine tools at the Technical University of Aachen. His current research interests include sensorless electromagnetic motor/actuator technologies, real-time signal processing and estimation techniques, precision multiaxis motion control, and ac/dc drive and high-precision machine control technologies. He has authored more than 160 published technical papers and is the holder of 16 patents, with two more pending.

Dr. Lorenz was the IEEE Industry Applications Society (IAS) President for 2001, a Distinguished Lecturer of the IAS for 2000/2001, immediate past Chair of the IAS Awards Department, and past Chairman of the IAS Industrial Drives Committee, and is a member of the IAS Industrial Drives, Electric Machines, Industrial Power Converter, and Industrial Automation and Control Committees. $\mathrm{He}$ is also the current Chair of the Periodicals Committee for the IEEE Technical Activities Board. He is a member of the IEEE Sensor Council AdCom and the IEEE Neural Network AdCom. He is a Registered Professional Engineer in the States of New York and Wisconsin. He is also a member of the American Society of Mechanical Engineers, Instrument Society of America, and Society of PhotoOptical Instrumentation Engineers. He has won 15 prize paper awards. 